\title{
Korrelation der Konzentrations- und Druckabhängigkeit des Wärmeübergangskoeffizienten beim Sieden von binären Stoffgemischen aus Aceton und Ethanol
}

\author{
Josef Schmadl und Romel Wittl
}

\section{Problematik}

Bislang gibt es keine einheitliche Theorie, um den Wärmeibbergang beim Blasensieden in freier Konvektion für binäre Gemische ohne experimentelle Daten und mit ausreichender Genauigkeit vorauszuberechnen. In der Literatur gibt es jedoch zahlreiche Korrelationsgleichungen bzw. Modelle für die praktische Berechnung des Wärmeübergangskoeffizienten (WÜK) [1]. Diese Modelle oder Gleichungen sind empirische oder halbempirische Ansätze, die nur auf spezielle Stoffpare anwendbar oder auf niedrige Siededrücke beschränkt sind oder nicht alle Einflussparameter enthalten. Die bislang in der Literatur vorgeschlagenen Berechnungsgleichungen lassen sich im Wesentlichen auf einige Grundtypen zurückführen (siehe Symbole und Abkürzungen im Anhang):

Typ I: $\quad \frac{\Delta T_{m i x}}{\Delta T_{i d}}=f\left(\bar{y}_{1}-\bar{x}_{1}\right)$

mit $\Delta T_{i d}$ als molanteilig gewichteter Mittelwert der Wanduiberhitzungen der reinen Stoffe und f einer algebraischen empirischen oder halbempirischen Funktion mit der Konzentrationsdifferenz zwischen Dampf und siedender Flüssigkeit $\bar{y}_{1}-\bar{x}_{1}$ als Variable.

Eine Gleichung diesen Typs ist die Gleichung von Stephan und Körner [2], die auch im VDI-Wärmeatlas [3] empfohlen wird und im Folgenden Gegenstand dieser Arbeit ist. In späteren Arbeiten wurde anstelle der reduzierten, dimensionslosen Wandüberhitzung die äquivalente Größe direkt mit den Wärmeüibergangskoeffizienten gebildet:

Typ II: $\quad \frac{\alpha_{m i x}}{\alpha_{i d}}=f\left(\bar{y}_{1}-\bar{x}_{1}\right)$

mit $\alpha_{i d}$ als molanteilig gewichteter Mittelwert der WüKen der reinen Stoffe. Beispiele für solche Gleichungen sind z. B. die von Stephan und Happel [4], Bier und Schmadl [5] und die im VDI-Wärmeatlas empfohlene Gleichung von Schliinder [3].

Einige neuere Gleichungen, z. B. die von Fujita und Tsutsui [1], verwenden die Differenz zwischen Tau- und Siedepunkt $T_{d}-T_{b}$ anstelle der Konzentrationsdifferenz $\bar{y}_{1}-\bar{x}_{1}$ :

Typ III: $\quad \frac{\alpha_{m i x}}{\alpha_{i d}}=f\left(T_{d}-T_{b}\right)$
In dieser Arbeit sollen anhand von eigenen Messdaten, die in einer vorherigen Arbeit beschrieben sind [6], folgende zwei Fragestellungen untersucht werden:

- Wie zuverlässig ist die Gleichung von Stephan und Körner für das Stoffpaar Aceton/Ethanol anwendbar?

- Wie gut lassen sich die eigenen Messwerte mit dieser Gleichung reproduzieren, wenn anstelle der Konzentrationsdifferenz $\bar{y}_{1}-\bar{x}_{1}$ die Temperaturdifferenz $T_{d}-T_{b}$ eingesetzt wird?

\section{Die Gleichung von Stephan und Körner}

Stephan und Körner [1] fanden bereits 1967 eine Beziehung zwischen der Wanduiberhitzung gegenuiber der siedenden Mischung $\Delta T$ und der Konzentrationsdifferenz zwischen dampfförmiger und flüssiger Phase $\bar{y}_{1}-\bar{x}_{1}$ von der Form:

$$
\frac{\Delta T_{m i x}}{\Delta T_{i d}}=1+C .\left|\bar{y}_{1}-\bar{x}_{1}\right|
$$

Der Index „mix“ wird im Folgenden aus Einfachheitsgründen weggelassen und der $\Delta T_{\text {id }}$ entsprechende WüK mit $\hat{\alpha}$ bezeichnet. Die Konstante $\mathrm{C}$ ist eine von Stoffsystem und Druck abhängige, lineare Druckfunktion. Mit dem definitionsgemäßen Zusammenhang zwischen Wandüberhitzung, Wärmestromdichte und Wärmeübergangskoeffizient

$$
\hat{\alpha}=\frac{\dot{q}}{\Delta T_{i d}}
$$

bekommt die Gleichung dann die Form

$$
\frac{\alpha}{\hat{\alpha}}=\frac{1}{1+A_{0} \cdot(0,88+0,12 \cdot p / \text { bar }) \cdot\left|\bar{y}_{1}-\bar{x}_{1}\right|}
$$

mit $A_{0}$ als stoffpaarspezifischem Parameter. Die Autoren geben an, dass bei Anwendung dieser Gleichung die eigenen Messwerte im Druckbereich von 1 bis 10 bar mit einem mittleren quadratischen Fehler von etwa $\pm 8 \%$ wiedergegeben werden. Diese Gleichung wird im VDIWärmeatlas als Berechnungsgrundlage empfohlen.

Darin nicht enthalten ist der Einfluss der Wärmestromdichte, was die Verwendbarkeit der Gleichung auf eine einzige Wärmestromdichte, nämlich $\dot{q}=100 \mathrm{~kW} / \mathrm{m}^{2}$, beschränkt. Andere Autoren haben später auch den Einfluss der Wärmestromdichte berüicksichtigt, z. B. Jung- 
nickel [7] und Schlünder [3]. Die Gleichung von Jungnickel hat sich aber bei Anwendung auf fremde Messergebnisse nicht bewährt. Für die Gleichung von Schlünder sind Stoffdaten erforderlich, die nicht ohne weiteres zugänglich sind, z. B. der Stoffuibertragungskoeffizient, was die Anwendung erschwert.

Für das hier betrachtete Stoffpaar Aceton-Ethanol gibt Körner eine Konstante $A_{0}$ von 0,75 an, die durch Anpassung an damals verfuigbare, fremde Messergebnisse erhalten worden war. Allerdings gilt diese Konstante nach Angabe der Autoren nur für einen Druck von $1 \mathrm{bar}$, womit die Gültigkeit der linearen Druckfunktion in Gleichung (Gl.) [6] fuir dieses Stoffpaar zumindest unsicher ist. Diese Einschränkung sowie auch die nicht ausreichende Literaturdatenbasis für das untersuchte Stoffpaar waren Anlass, die Gleichung unter Verwendung des eigenen, auf Druicke bis 10 bar erweiterten Datenmaterials zu iiberprïifen.

\section{Thermodynamische Daten des Dampf/Flüssig-Gleichgewichts}

Im Hinblick auf die korrelative Modellierung des Wärmeiibergangskoeffizienten stellt sich nun die Frage, ob dafür die Konzentrationsdifferenz $\bar{y}_{1}-\bar{x}_{1}$ oder die Differenz von Tau- und Siedetemperatur $T_{d}-T_{b}$ die geeignetere Größe ist. $\bar{y}_{1}-\bar{x}_{1}$ stellt die Breite, $T_{d}-T_{b}$ die Höhe der Siedelinsenaufspreizung dar (siehe [6], Abb. 1). In früheren Untersuchungen wurde ausschließlich $\bar{y}_{1}-\bar{x}_{1}$ für Korrelationszwecke verwendet. In der neueren Literatur taucht dabei öfter $T_{d}-T_{b}$ auf [1]. In Abbildung 1a und $1 \mathrm{~b}$ sind beide Größen einander gegenuibergestellt und mit Literaturangaben und eigenen Berechnungen verglichen. Abbildung 1a enthält eigene NRTL- und UNIFAC-Berechnungen fuir die untersuchten Druicke, eigene gaschromatographisch ermittelte Messwerte sowie Vergleichswerte von Alpay und Hakan [7].

Die NRTL- und UNIFAC-Berechnungen weichen bis zu ca. $6 \mathrm{Mol}-\%$ bei 1 bar von einander ab. Die Übereinstimmung der eigenen Messwerte mit diesen Berechnungen ist relativ gut, außer am linken Konzentrationsrand, wo es wegen der in [6] beschriebenen Messunsicherheit bezuiglich $\bar{y}_{1}$ und des steilen Kurvenanstiegs zu Abweichungen bis zu ca. 10,5 Mol-\% kommt. Trotzdem stimmen die Messwerte von Alpay et al. bei 4 bar erstaunlich gut mit diesen Messungen überein. Sie weichen auch in dem fraglichen Konzentrationsbereich um höchstens 1 Mol-\% davon ab. Auch die eigenen Messwerte, weniger deutlich die von Alpay et al., deuten besonders bei 7 und 10 bar auf das Vorhandensein eines azeotropen Punktes hin, was in [6] diskutiert worden ist.

In Abb.1b sind die Differenzen zwischen Tau- und Siedetemperatur den Konzentrationsdifferenzen gegenübergestellt. Prinzipiell ergibt sich natürlich ein ähnlicher Sachverhalt, d. h. $T_{d}-T_{b}$ weist in etwa demselben mittleren Konzentrationsbereich bei konstantem Druck ein Maximum auf wie auch $\bar{y}_{1}-\bar{x}_{1}$, das mit steigendem Druck abnimmt. Am rechten Konzentrationsrand stellt sich der Bereich um den azeotropen Punkt allerdings quantitativ unterschiedlich dar: Während die Konzentrationsdifferenz z. B. bei $p=10$ bar einen Nullwert bei etwa $\bar{x}_{1}=0,75$ aufweist und dann im Bereich $0,75<\bar{x}_{1}<1$ wieder von Null verschieden ist, bleibt die Temperaturdifferenz in diesem Konzentrationsbereich nahe Null, was an der besonderen Form der Siedlinse im Falle azeotroper Stoffsysteme liegt. Von daher ist zu erwarten, dass sich Beschreibung der relativen Verschlechterung des WÜKen $\alpha / \hat{\alpha}$ mit den beiden Größen $\bar{y}_{1}-\bar{x}_{1}$ und $T_{d}-T_{b}$ unterschiedlich gut gelingt.
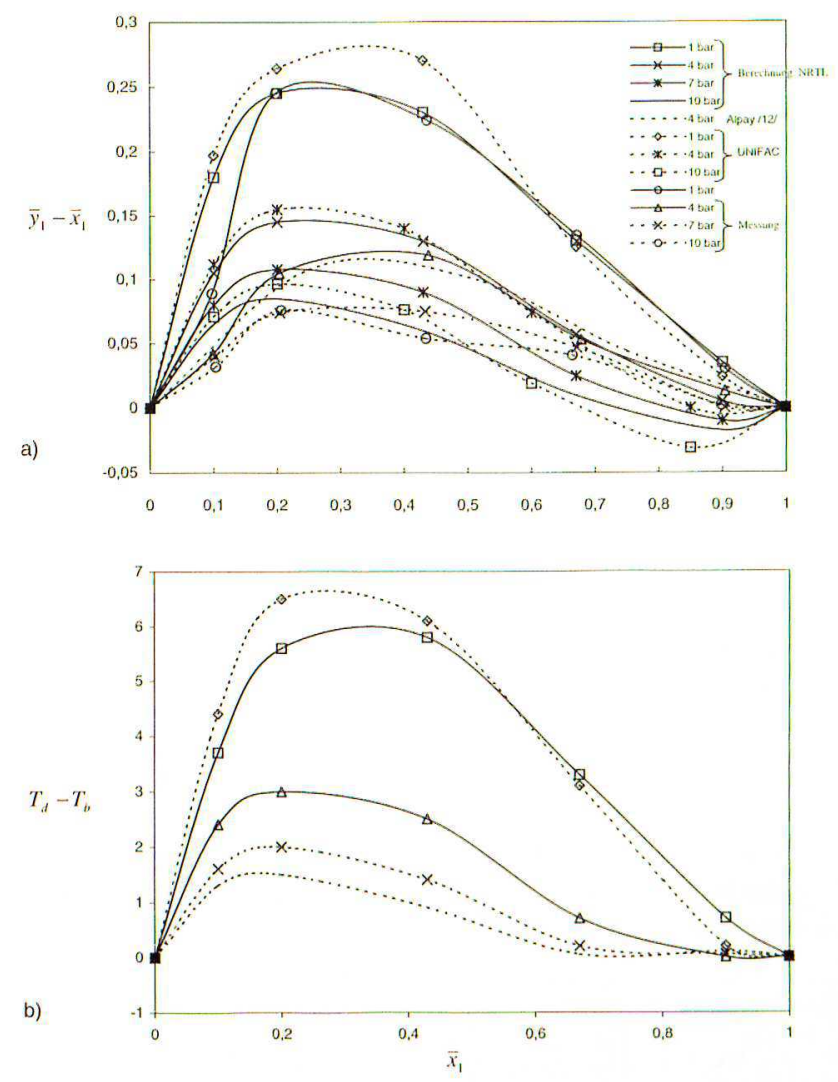

Abb. 1: Konzentrationsdifferenz $\bar{y}_{1}-\bar{x}_{1}$ und Differenz $T_{d}-T_{b}$ zwischen Tau- und Siedetemperatur in Abhängigkeit vom Molenbruch bei den untersuchten Drücken.

\section{Korrelation der relativen Verschlechte- rung des Wärmeübergangs $\alpha / \hat{\alpha}$ mit der Konzentrationsdifferenz $\left|\bar{y}_{1}-\bar{x}_{1}\right|$}

Erfahrungsgemäß tritt bei konstantem Druck die stärkste Verschlechterung des Wärmeuibergangs in etwa in dem Konzentrationsbereich auf, in welchem auch die Konzentrationsdifferenz $\bar{y}_{1}-\bar{x}_{1}$ ein Maximum durchläuft. Vergleicht man die Lage des Maximums der Konzentrationsdifferenz $\left(\bar{y}_{1}-\bar{x}_{1}\right)_{\max }$ mit der des Minimums des reduzierten Wärmeübergangskoeffizienten $\left(\alpha / \alpha_{i d}\right)_{\min }$, stellt man fest, dass die beiden Extremwerte gegeneinander leicht verschoben sind. Allerdings bleibt diese Verschiebung im Rahmen der in [6] diskutierten Unschärfe von $\Delta \bar{x}_{1}$. Im Folgenden wird versucht, die Messergebnisse des untersuchten Stoffpaares mit der Gleichung von Stephan und Körner wiederzugeben. In Abb. 2 sind die experimentellen Ergebnisse sowie die mit Gl. (6) und $A_{0}=0,75$ gerechneten Daten dargestellt. 


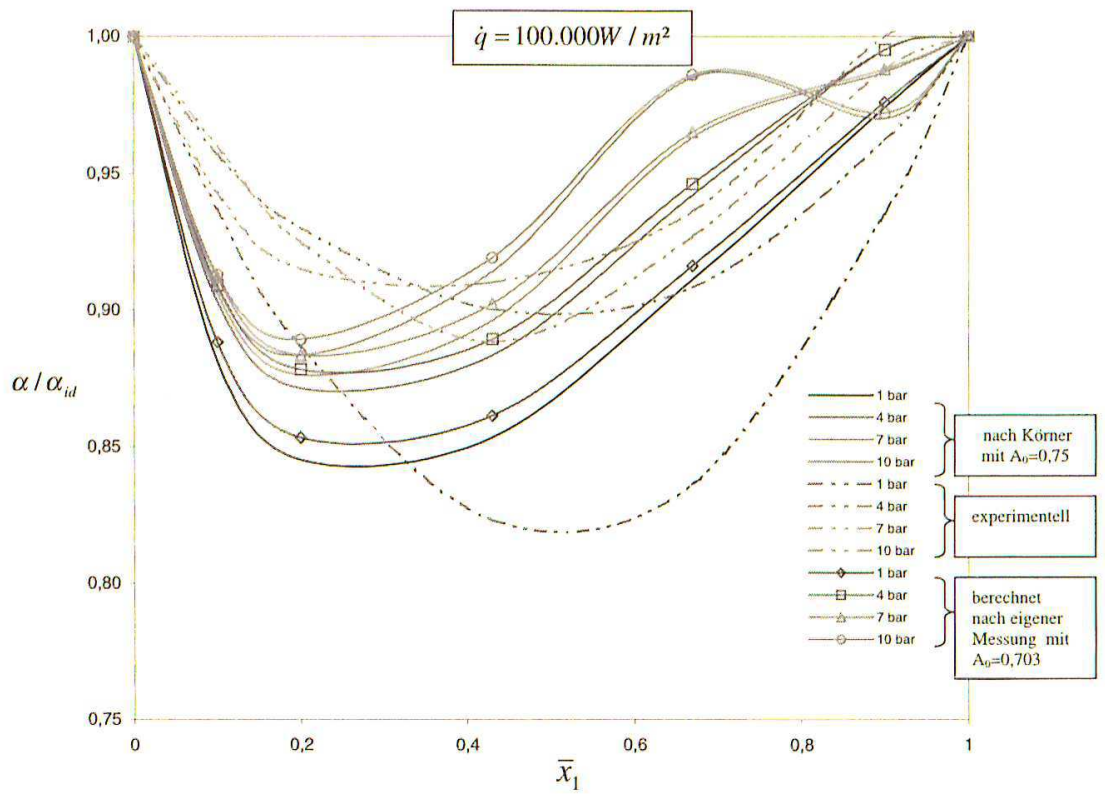

Abb. 2: reduzierter Wärmeübergangskoeffizient $\alpha / \hat{\alpha}$ in Abhängigkeit vorn Aceton-Molenbruch $\bar{x}_{1}$ bei konstanter Wärmestromdichte $100 \mathrm{~kW} / \mathrm{m}^{2}$ und verschiedenen Siededrücken. Die gestrichelten Kurven stellen die experimentellen Messergebnisse dar, die durchgezogenen Kurven die gerechneten Daten nach Körner mit $A_{0}=0,75$. Die durchgezogenen Kurven mit Symbolen stellen die in dieser Arbeit gerechneten Daten mit der in dieser Arbeit ermittelten Konstante $A_{0}=0,70 \mathrm{dar}$ :

In Tabelle 1 ist die prozentuale Abweichung der mit Gl. (6) gerechneten von den experimentellen Daten im Kurvenminimum $\varepsilon$ sowie die Verschiebung der Konzentrationen der beiden entsprechenden Kurven im Minimum $\Delta \bar{x}_{1}$ dargestellt. Beim reduzierten Wärmeübergangskoeffizienten ergibt sich eine Abweichung von 1,45 bis zu 3,36\% zwischen experimentellen und errechneten Werten. Man sieht, dass die Konzentrationen des Minimums bei allen Drïcken zwischen Rechnung und Messung um 16,5 Mol-\% bis 30 Mol-\% verschoben sind.

\begin{tabular}{|l|l|l|l|l|}
\hline Druck [bar] & 1 & 4 & 7 & 10 \\
\hline$\varepsilon[\%]$ & 2,98 & 3,36 & 1,45 & 2,82 \\
\hline$\Delta \bar{x}_{1}$ & 0,24 & 0,30 & 0,23 & 0,165 \\
\hline
\end{tabular}

Tab. 1: Zusammenfassung des prozentualen Unterschieds zwischen experimentellen und mit $A_{0}=0,75$ gerechneten Daten des reduzierten Wärmeübergangskoeffizienten im Minimum und der Verschiebung der Konzentrationen der beiden Darstellungen im Minimum (Abb. 2)

mit

$$
\varepsilon=\frac{\left(\frac{\alpha}{\hat{\alpha}}\right)_{\text {exp,min }}-\left(\frac{\alpha}{\hat{\alpha}}\right)_{\text {calc,min }}}{\left(\frac{\alpha}{\hat{\alpha}}\right)_{\text {exp, min }}} .100 \%
$$

und

$$
\Delta \bar{x}_{1}=\bar{x}_{1, \exp , \min }-\bar{x}_{1, \text { calc, } \min }
$$

Zwecks Überprüfung von Gl. (6) wurde nun in dem durch die eigenen Messwerte erweiterten Druckbereich $1<p<10$ bar eine neue Konstante $A_{0}$ ermittelt, da seinerzeit Körner für seine Korrelationsrechnungen keine Messwerte bei Drüicken über 5 bar zur Verfügung gestanden hatten. Für diesen Zweck wurden Einzelwerte von $A_{0}$ über Gl. (6) durch Anpassung an die eigenen Messwerte berechnet, wobei die Konzentrationsdifferenz $\bar{y}_{1}-\bar{x}_{1}$ jeweils aus der mit NRTL gerechneten Siedelinse entnommen wurden. Sie sind in der Tabelle 2 enthalten.
Es ist zunächst keine systematische Druckabhängigkeit dieser Rechenwerte erkennbar. Bei konstantem Druck beobachtet man allerdings, dass die $A_{0}$-Werte mit steigender Konzentration der leichter Siedenden in der Fluissigkeit leicht ansteigen. Das Ergebnis dieser Berechnung ist ein mittlerer $A_{0}$-Wert von 0,70 . Für die Mittelwertbildung wurden die in Tabelle 8 fett eingerahmten schraffierten Einzelwerte nicht berücksichtigt, weil die $\mathrm{A}_{0}$ Werte bei sehr kleinen $\bar{y}_{1}-\bar{x}_{1}$-Werten nach Gl. (6) gegen $\infty$ gehen, d. h. sinnlos werden. Alle anderen $A_{0}$-Werte aus Tabelle 8 wurden arithmetisch gemittelt. Mit diesem $A_{0}$-Wert ergeben sich die in Abb. 2 fett eingezeichneten Isobaren.

Man sieht, dass sich damit geringfügig schwächere Verschlechterungen errechnen lassen als mit dem Körner-Wert $A_{0}=0,75$. Ansonsten ist der Kurvenverlauf qualitativ völlig analog. Die maximalen relativen Abweichungen $\varepsilon$ und Konzentrationsverschiebungen des Minimums $\Delta \bar{x}_{1}$ zwischen Messung und Rechnung mit $A_{0}=0,70$ sind in der Tab. 3 analog zu Tab. 1 dargestellt. Für den reduzierten Wärmeübergangskoeffizienten ergibt sich demnach ein prozentualer Unterschied von 0,73 bis zu 4,1\% zwischen den experimentellen und den errechneten Werten im untersuchten Druckbereich. Man sieht, dass mit $A_{0}=0,70$ die Isobare $p=1$ bar schlechter beschrieben wird als mit $A_{0}=0,75$. Jedoch werden die Isobaren 4,7 und 10 bar besser wiedergegeben. Die Konzentrationsverschiebungen $\Delta \bar{x}_{1}$ in Tab. 3 unterscheiden sich nicht wesentlich von jenen in Tab. 1. Als Resümee aus diesen Berechnungen geht hervor, dass die Messwerte mit dem der ursprünglichen $A_{0}$-Wert von Körner $A_{0}=0,75$ insgesamt etwas schlechter wiedergegeben werden, bei 1 bar besser, bei höheren Drücken aber schlechter als mit $A_{0}=$ 0,75, womit die von Körner selbst erwähnte Unsicherheit bezüglich der Gültigkeit des $A_{0}$-Wertes 0,75 bei Drü-

\begin{tabular}{|c|c|c|c|c|c|}
\hline \multirow[b]{2}{*}{ Druck [bar] } & \multicolumn{5}{|c|}{$\begin{array}{l}\text { Die untersuchten Konzentrationen des Gemisches Aceton- } \\
\text { Ethanol }\end{array}$} \\
\hline & $\bar{x}_{1}=0,1$ & $\bar{x}_{1}=0,2$ & $\bar{x}_{1}=0,43$ & $\vec{x}_{1}=0,67$ & $\vec{x}_{1}=0,9$ \\
\hline 1 & 0,374 & 0,525 & 0,935 & 1,509 & 1,986 \\
\hline 4 & 0,315 & 0,382 & 0,628 & 1,242 & 5,809 \\
\hline 7 & 0,303 & 0,429 & 0,815 & 1,526 & 0,706 \\
\hline 10 & 0,447 & 0,525 & 0,792 & 3,287 & 0 \\
\hline
\end{tabular}
cken $p>1$ bar bestätigt wird.

Tab. 2: Zusammenfassung der gerechneten $A_{0}$-Werte aus den mit Gl. (6) korrelierten eigenen Messwerten.

\begin{tabular}{|l|l|l|l|l|}
\hline Druck [bar] & 1 & 4 & 7 & 10 \\
\hline$\varepsilon[\%]$ & 4,1 & 2,29 & 0,73 & 2,23 \\
\hline$\Delta \bar{r}_{1}$ & 0,254 & 0,252 & 0,207 & 0,162 \\
\hline
\end{tabular}

Tab. 3: Zusammenfassung der Fehler zwischen experimentellen und mit $A_{0}=0,70$ gerechneten Werten des reduzierten Wärmeübergangskoeffizienten und der Verschiebung der Konzentrationen der beiden Darstellungen im Minimum. 


\section{Korrelation der relativen Verschlechte- rung des Wärmeübergangs $\alpha / \hat{\alpha}$ mit der Differenz zwischen Tau- und Siede- temperatur und $T_{d}-T_{b}$}

Ersetzt man in Gl. (6) $\bar{y}_{1}-\bar{x}_{1}$ durch $T_{d}-T_{b}$, erhält man:

$$
\frac{\alpha}{\hat{\alpha}}=\frac{1}{1+A_{0, T} \cdot(0,88+0,12 \cdot p / \text { bar }) \cdot\left|T_{d}-T_{b}\right|}
$$

$T_{d}-T_{b}$ ist die Differenz zwischen Tautemperatur des Dampfes und Siedetemperatur der Fliissigkeit im Pool aus Abb. 1. Mit Gl. (9) ergibt sich naturgemäß ein anderes $A_{0}$, hier $A_{0, T}$ genannt. In Tab. 4 sind die mit den eigenen Messwerten und Gl. (9) errechneten Einzelwerte fuir $A_{0, T}$ enthalten, analog zu Tab. 2 . Auch hier wurden die fuir die Mittelwertbildung unsinnigen $A_{0, T}$-Werte bei $\alpha / \hat{\alpha} \cong 1$ (schraffiert in Tabelle 10) weggelassen. Im Ergebnis ergab sich als Mittelwert $A_{0, T}=0,039$.

\begin{tabular}{|l|l|l|l|l|l|}
\hline \multirow{4}{*}{ Druck [bar] } & \multicolumn{5}{|l|}{$\begin{array}{l}\text { Die untersuchten Konzentrationen des Gemisches Aceton- } \\
\text { Ethanol }\end{array}$} \\
\cline { 2 - 6 } & $\vec{x}_{1}=0,1$ & $\vec{x}_{1}=0,2$ & $\vec{x}_{1}=0,43$ & $\vec{x}_{1}=0,67$ & $\vec{x}_{1}=0,9$ \\
\hline 1 & 0,015 & 0,019 & 0,0358 & 0,0633 & 0,116 \\
\hline 4 & 0,0157 & 0,0179 & 0,033 & 0,1034 & 0,415 \\
\hline 7 & 0,0147 & 0,0219 & 0,0506 & 0,305 & 0,282 \\
\hline 10 & 0,0196 & 0,0263 & 0,0553 & 1,644 & 0,003 \\
\hline
\end{tabular}

Tab. 4: Zusammenfassung der $A_{0, T}$-Einzelwerte, wie sie aus den eigenen Messdaten mit Gl. (9) errechnet wurden

In Abb. 3a ist die mit Gl. (9) und $A_{0, T}=0,039$ berechnete relative Verschlechterung des Wärmeuibergangs $\alpha / \hat{\alpha}$ in Abhängigkeit vom Molenbruch des leichter Siedenden in der Fliissigkeit bei konstanter Wärmestromdichte $\dot{q}=100 \mathrm{~kW} / \mathrm{m}^{2}$ und verschiedenen Drücken vergleichsweise zu den eigenen Messwerten dargestellt. In Abb. 3b und 3c sind zum Vergleich die analogen, mit Gl. (6) und $A_{0}=0,70$ bzw. mit Gl. (6) und $A_{0}=0,75$ gerechneten Daten enthalten. Man sieht, dass alle drei dargestellten Korrelationen einen qualitativ fast gleichen Kurvenverlauf zeigen, wobei in der Abb. 3a die relative Verschlechterung des Wärmeübergangs geringfügig stärker ausfällt. In der Tab. 5 ist die maximale Abweichung $\varepsilon$ und Konzentrationsverschiebungen des Minimums $\Delta \bar{x}_{1}$ analog zu den Tabellen 1 und 3 dargestellt.

\begin{tabular}{|l|l|l|l|l|}
\hline Druck [bar] & 1 & 4 & 7 & 10 \\
\hline$\varepsilon[\%]$ & 3,29 & 5,31 & 1,36 & 4,06 \\
\hline$\Delta \bar{x}_{1}$ & 0,253 & 0,269 & 0,263 & 0,186 \\
\hline
\end{tabular}

Tab. 5: Zusammenfassung der prozentualen Unterschiede zwischen experimentellen und mit Gl. (9) und $A_{o, T}=0,039$ gerechneten Werten des reduzierten Wärmeübergangskoeffizienten E nach Gl. (7) und der Verschiebungen des Minimums der beiden Darstellungen $\Delta \bar{x}_{1}$ nach Gl. (8).

Die prozentualen Abweichungen $\varepsilon$ in der Tab. 5 sind mit $1,36 \%$ bis 5,31\% von der gleichen Größenordnung wie die in Tab. 1 und 3 enthaltenen Werte. Auch die Konzentrationsverschiebungen $\Delta \bar{x}_{1}$ sind mit Werten zwischen 18,6 Mol-\% und 26,9 Mol-\% ähnlich den Vergleichswerten. Dies bedeutet, dass sich Konzentrationsdifferenz $\bar{y}_{1}-\bar{x}_{1}$ und Temperaturdifferenz $T_{d}-T_{b}$ in Gl. (6) bzw. (9) ohne weiteres austauschen lassen bei entsprechender Umrechnung von $A_{0}$. Dabei wird die relative Verschlechterung des Wärmeuibergangs sowohl bezüglich der qua- litativen Konzentrationsabhängigkeit $\alpha / \hat{\alpha}=f\left(\bar{x}_{1}\right)$ bei $p=$ konstant bzw. bezüglich der qualitativen Druckabhängigkeit $\alpha / \hat{\alpha}=f(p)$ bei $\bar{x}_{1}=$ konstant, als auch beziiglich der quantitativen Rechenwerte $\alpha / \hat{\alpha}$ im Rahmen der Messwertegenauigkeit dieser Arbeit in etwa gleich gut wiedergegeben. Die bei der Anwendung von Gl. (6) beobachtete Verschiebung der $\alpha / \hat{\alpha}$-Minima $\Delta \bar{x}_{1}$ nach Gleichung (8) wird bei Verwendung von Gl. (9) mit $T_{d}-T_{b}$ anstelle von $\bar{y}_{1}-\bar{x}_{1}$ nicht beseitigt. Ob sich dieser Befund generell auch bei anderen Stoffsystemen bestätigt, müsste durch weitere Untersuchungen geklärt werden.

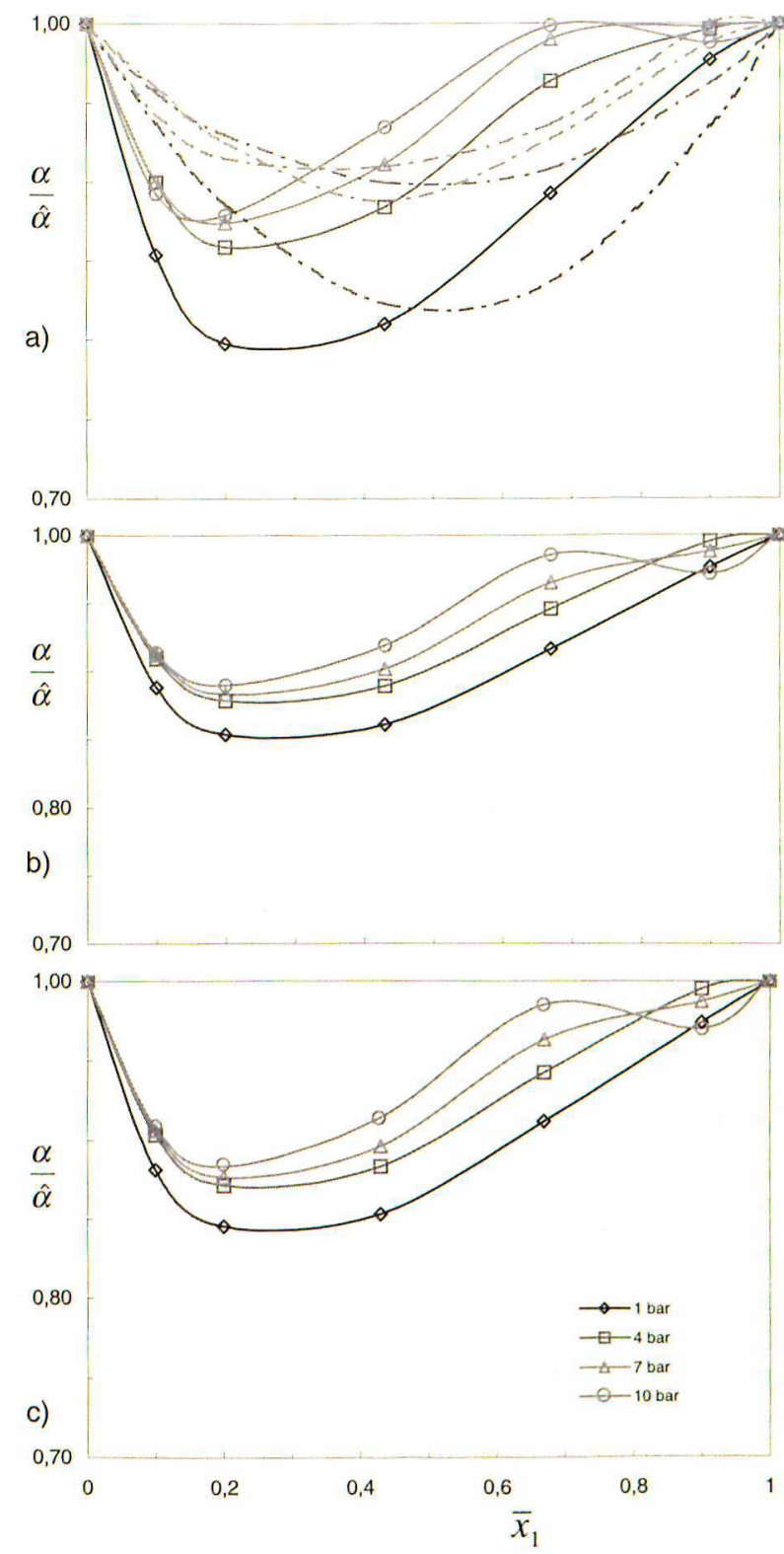

Abb. 3: Reduzierter Wärmeübergangskoeffizient $\alpha / \hat{\alpha}$ in Abhängigkeit vom Aceton-Molenbruch der siedenden Flüssigkeit $\bar{x}_{1}$ bei konstanter Wärmestromdichte $100.000 \mathrm{~W} / \mathrm{m}^{2}$ und verschiedenen Siededrücken. a) Die eingezeichneten Punkte sind gerechnet mit Gl. (9) und $A_{0}=0,039$. Vergleichsweise dazu sind die experimentellen Daten eingezeichnet. b) Die eingezeichneten Punkte sind gerechnet mit Gl. (6) und $A_{0}=0,70$. c) Die eingezeichneten Punkte sind gerechnet mit Gl. (6) und $A_{0}=0,75$. 


\section{Zusammenfassung}

Setzt man in Gl. (6) die von Körner vorgeschlagene Konstante $A_{0}$, die mit NRTL gerechneten Konzentrationsdifferenzen $\bar{y}_{1}-\bar{x}_{1}$ und die jeweiligen Drïcke ein, so lassen sich die Messergebnisse in der Form $\alpha / \hat{\alpha}$ mit einer Abweichung von ca. $3 \%$, ermittelt jeweils im Minimum von $\alpha / \hat{\alpha}$, wiedergeben. Allerdings liegen dabei die gemessenen und nach Gl. (6) gerechneten Minima von $\alpha / \hat{\alpha}$ bei allen Drücken um ca. 16 bis 30 Mol- $\%$ auseinander. Da die von Körner angegebene Konstante $A_{0}=0,75$ für das untersuchte Stoffpaar Aceton-Ethanol nur für den Druck $p=1$ bar guiltig ist, wurde mit den eigenen Messwerten die Konstante $A_{0}=0,70$ ermittelt, die bis 10 bar guiltig ist. Um eine Verringerung der erwähnten Minimaverschiebung von $\alpha / \hat{\alpha}$ zu erzielen, wurde außerdem versucht, in Gl. (6) die Konzentrationsdifferenz $\bar{y}_{1}-\bar{x}_{1}$ durch die Differenz der Tau- und Siedetemperatur zu ersetzen und damit eine andere neue Konstante $A_{0, T}$ zu ermitteln (Gl. 9). So wurde durch eine analoge Anpassung an die Messwerte eine Konstante $A_{0, T}=0,039$ ermittelt. Die Korrelation der Messwerte mit Gl. (9) und $A_{0, T}=0,039$ zeigt, dass die dabei auftretenden Abweichungen der Rechenwerte von den Messwerten die gleiche Größenordnung haben wie bei Anwendung der ursprïnglichen Gl. (6), d. h. auch mit $T_{d}-T_{b}$ anstelle von $\bar{y}_{1}-\bar{x}_{1}$ verbessert sich die Minimaverschiebung nicht.

Bei der Anwendung von Gl. (6) aus /1/ auf Drücke $>1$ bar wird abweichend von $/ 1 /$ die Verwendung des in dieser Arbeit ermittelten Wertes $A_{0}=0,70$ für das Stoffsystem Aceton/Ethanol empfohlen, damit ein größerer Druckbereich abgedeckt wird. Mit vergleichbaren Ergebnissen kann aber auch die in dieser Arbeit modifizierte Gl. (9) mit $A_{0, T}=0,039$ angewendet werden, falls Tau- und Siedepunkt anstelle von Dampf- und Flïssigkeitskonzentration der Mischung bekannt sind.

\section{Literatur}

[1] D. GORENFLO and R. KÖSTER: Pool Boiling Heat Transfer from horizontal Tubes to Mixtures. Laboratorium für Wärme- und Kältetechnik. Universität (GH) Paderborn, Germany

[2] VDI-WÄRMEATLAS: VDI-Verlag Duisseldorf, 7. Auflage, Abschnitt Hab, 1994

[3] K. STEPHAN und M. KÖRNER: Berechnung des Wärmeübergangs verdampfender binärer Fliissigkeitsgemische. Chem. Ing. Technik 41 (7), S. 409-416, 1969

[4] K. STEPHAN und O. HAPPEL: Heat Transfer from Nucleate to the Beginning of Film Boiling in Binary Mixtures, Proc. 5th Int. Heat Transfer Conf., Tokio, Bd. 4, 1974.

[5] K. BIER, J. SCHMADL und D. GORENFLO: Wärmeübergang beim Blasensieden von Zweistoffgemischen unter höheren Drïcken. DKV-Tagungsbericht 9. 1982.

[6] J. SCHMADL und R. WITTL: Experimentelle Untersuchung des Wärmeüibergangs beim Sieden binärer Stoffgemische aus Aceton und Ethanol. Wissenschaftliche Beiträge TFH Wildau, Heft 2002.
[7] H. ALPAY and F. BALKAN: Nucleate pool boiling performance of acetone-ethanol and methylenechlorideethanol binary mixtures, Department of Chemical Engineering, Ege University, Izmir, Turkey, 1988-1989.

\section{Symbole und Abkürzungen}

$\begin{array}{ll}\alpha & \text { Wärmeübergangskoeffizient }\left[/\left(\mathrm{m}^{2} . \mathrm{K}\right)\right] \\ \alpha_{i d} & \begin{array}{l}\text { molanteilig gemittelter Wärmeübergangsko- } \\ \text { effizient der reinen Komponenten }\end{array} \\ \Delta T_{i d} & \begin{array}{l}\text {,ideale“ Wandüberhitzung als molanteilig ge- } \\ \text { wichteter Mittelwert der entsprechenden Rein- } \\ \text { stoffwerte }\end{array} \\ \bar{y}_{1}-\bar{x}_{1} & \begin{array}{l}\text { Konzentrationsdifferenz zwischen Dampf und } \\ \text { siedender Flïssigkeit }\end{array} \\ \alpha / \hat{\alpha} & \text { reduzierter Wärmeübergangskoeffizient } \\ \text { Index „mix“ } & \text { Stoffwert der Mischung }\end{array}$

\section{Autoren}

Prof. Dr.-Ing. Josef Schmadl

Dipl.-Ing. (grad.) Romel Wittl

Technische Fachhochschule Wildau

Technikum fuir Thermische Verfahrenstechnik

Tel. (0 33 75) 508-110, Fax (0 33 75) 508-906

E-Mail: jschmadl@igw.tfh-wildau.de 